\title{
Antibody-mediated FOXP3 protein therapy induces apoptosis in cancer cells in vitro and inhibits metastasis in vivo
}

\author{
EMIL HEINZE ${ }^{1,2}$, SCOTT BALDWIN $^{3}$, GRACE CHAN $^{1}$, JAMES HANSEN $^{1}$, JASON SONG $^{1}$, \\ DOUGLAS CLEMENTS ${ }^{1}$, ROBERT ARAGON ${ }^{3}$, ROBERT NISHIMURA ${ }^{1}$, \\ MARK REEVES ${ }^{3}$ and RICHARD WEISBART ${ }^{1}$ \\ ${ }^{1}$ Veterans Affairs Greater Los Angeles Health Care System, Sepulveda; ${ }^{2}$ Olive View-University of California at \\ Los Angeles Medical Center, Sylmar; ${ }^{3}$ Loma Linda Veterans Affairs Medical Center, Loma Linda, CA, USA
}

Received December 10, 2008; Accepted February 25, 2009

DOI: 10.3892/ijo_00000325

\begin{abstract}
In addition to its immune suppressive function in T-regulatory cells, the nuclear transcription factor, FOXP3, has been identified as a tumor suppressor. To evaluate the clinical efficacy of monoclonal antibody (mAb) 3E10 Fv antibody-mediated FOXP3 protein therapy of cancer, the Fv-FOXP3 fusion protein produced in Pichia pastoris was tested on breast, ovarian, and colon cancer cells in vitro, and with colon cancer cells in vivo in a mouse model of colon cancer metastasis to liver. Treatment with Fv-FOXP3 resulted in dose-dependent cell death of cancer cells in vitro. Apoptosis was established as a mechanism of cell death by demonstrating increased production of the p17 activated fragment of caspase- 3 by cancer cells in response to Fv-FOXP3 and inhibition of cell killing by the caspase inhibitor, Z-VADFMK. Fv-FOXP3 treatment resulted in clinically significant reduction in tumor burden in a syngeneic model of colon cancer metastasis to liver in Balb/c mice. These results represent the first demonstration of effective full-length FOXP3 protein therapy and emphasize the clinical potential of mAb 3E10 as an intracellular and intranuclear delivery vehicle of FOXP3 for prevention and treatment of cancer metastasis.
\end{abstract}

\section{Introduction}

FOXP3 is an X-linked forkhead-family nuclear transcription factor associated with the immune suppressive function of T-regulatory cells. A single mutation in the FOXP3 gene leads to autoimmunity in scurfy mice and the immunodysregulation, polyendocrinopathy, enteropathy, X-linked

Correspondence to: Dr Richard H. Weisbart, Veterans Affairs Medical Center (111S), 16111 Plummer Street, Sepulveda, CA 91343, USA

E-mail: rweisbar@ucla.edu

Key words: antibodies, transcription factors, gene regulation, cytotoxicity, apoptosis
(IPEX) syndrome in human males due to the complete lack of functional T-regulatory cells (1-4). Zuo et al serendipitously observed that female mice heterozygous for the FOXP3 mutation developed a high incidence of breast and other cancers (5). Further study of the murine breast cancers showed that $100 \%$ of tumor tissue contained mutant FOXP3 and that transfection of wild-type FOXP3 cDNA into different breast cancer cell lines inhibited cell growth and induced cell death by apoptosis ( 5 and online supplement therein). FOXP3 was also shown to be deleted or mutated in the majority of human breast cancer tissues sampled, providing a strong impetus for examining FOXP3 as a potential therapy for human cancer (5).

Although gene therapy is one approach to restoring FOXP3 function, numerous technical challenges have yet to be overcome. Protein replacement therapy is an alternative to gene therapy, and our laboratory has previously explored the use of an antibody, mAb 3E10 and its Fv fragment that penetrate living cells as a vehicle to deliver nuclear transcription factors into cells (6). In order to transport FOXP3 protein into cancer cells, we produced a fusion protein consisting of the Fv fragment of mAb 3E10 and FOXP3. We now show that the Fv fragment of mAb 3E10 transports FOXP3 into cancer cells and induces cell death by apoptosis.

\section{Materials and methods}

Cell lines. Human cancer cell lines SKOV3 (ovarian), OVCAR-3 (ovarian), MDA-MB-231 (breast), and MCF-7 (breast) were obtained from the American Type Culture Collection (ATCC, Manassas, VA). The murine CT26 cell line (colorectal) was also obtained from ATCC.

Antibodies. Mouse monoclonal $\mathrm{IgG}_{1}$ to FOXP3 C-terminus (ab22510) was obtained from Abcam Inc. (Cambridge, MA). Antibodies from Santa Cruz Biotechnology (Santa Cruz, CA) included mouse monoclonal $\mathrm{IgG}_{1}$ to human FOXP3 (sc-56680), goat polyclonal IgG to human actin C-11 (sc-1615), goat polyclonal IgG to $\mathrm{N}$-terminus of activated caspase-3 p17 of human origin (sc-22139), and mouse anti-goat IgG-HRP (sc-2354). Amersham ECL anti-mouse IgG-HRP (NA931V) was obtained from GE Healthcare Ltd., UK. 
Construction of pPICZA-Fv-FOXP3 and pPICZA-FOXP3 recombinant plasmids. Human FOXP3 cDNA was amplified by PCR from plasmid 13250 (Addgene, Cambridge, MA) with the following primers: sense primer, 5'-GGATCCATGCCCA ACCCCAGGCCT-3'; anti-sense primer, 5'-CCGCGGTCAG GGGCCAGGTGTAGGG-3'. The flanking BamHI and SacII restriction sites were used to ligate the PCR fragment into a cassette in pPICZA containing the Fv fragment of mAb 3E10 with a C-terminal $\mathrm{His}_{6}$ tag to produce the plasmid $p P I C Z A$ $F v$-FOXP3. FOXP3 alone was produced as a control. FOXP3 cDNA was amplified by PCR with the sense primer, 5'-GAA TTCATGCATCACCATCACCATCACCCCAACCCCAGG CCTG-3'; and anti-sense primer, 3'-CCGCGGTCAGGG GCC-3' to produce FOXP3 containing an N-terminal $\mathrm{His}_{6}$ tag. The PCR fragment was ligated into the EcoRI and SacII sites of $p$ PICZA to produce the plasmid, pPICZA-FOXP3.

Expression and purification of Fv-FOXP 3 and FOXP3 recombinant proteins. pPICZA-Fv-FOXP3 and pPICZAFOXP3 were electroporated into the Pichia pastoris $\mathrm{X}-33$ strain, and colonies were selected with Zeocin (Invitrogen, Carlsbad, CA) and identified with anti-his ${ }_{6}$ antibodies (Qiagen Inc., Valencia, CA). X-33 cells were grown in baffled shaker flasks with buffered glycerol/methanol medium, and protein synthesis was induced with $0.5 \%$ methanol according to the manufacturer's protocol (EasySelect Pichia Expression Kit, Invitrogen). The cells were lysed by two passages through a French Cell Press at 20,000 lbs/in ${ }^{2}$, and recombinant protein was purified from cell pellets solubilized in $9 \mathrm{M}$ guanidine $\mathrm{HCl}$ and 2\% NP40 by immobilized metal ion affinity chromatography (IMAC) on Ni-NTA-Agarose (Qiagen). Bound protein was eluted in $50 \mathrm{mM} \mathrm{NaH} \mathrm{PO}_{4}$ containing $300 \mathrm{mM} \mathrm{NaCl}$, $500 \mathrm{mM}$ imidazole, and $25 \%$ glycerol. Samples of wash and eluted fractions were electrophoresed in 4-20\% gradient SDS-PAGE (NuSep Ltd., Frenchs Forest, Australia), and recombinant proteins were identified by Western blotting to nitrocellulose membranes developed with anti-FOXP3 antibodies followed by alkaline-phosphatase-conjugated goat antibodies to mouse IgG. Alkaline phosphatase activity was measured by the chromogenic substrate, nitroblue tetrazolium chloride/5-bromo-4-chloro-3-indolylphosphate p-toluidine salt. Proteins were identified in SDS-PAGE gels with GelCode Blue Stain Reagent (Pierce Chemical Co., Rockford, IL). Eluted protein was concentrated to $10 \mu \mathrm{g} / \mathrm{ml}$, reconstituted with fetal calf serum to $5 \%$, and exchange dialyzed 100-fold in 30,000 MWCO spin filters (Millipore Corp., Billerica, MA) against McCoy's medium (Mediatech, Inc., Herndon, VA) containing 5\% glycerol.

Cellular penetration of Fv-FOXP3 in vitro. Penetration of Fv-FOXP3 was evaluated after incubating different cell lines with purified recombinant Fv-FOXP3 for $1 \mathrm{~h}$ in 96-well plates at concentrations that were not cytotoxic within this period of time. Cells were washed with PBS, fixed with $100 \%$ ethanol, and Fv-FOXP3 was detected with anti-FOXP3 antibodies followed by alkaline-phosphatase-conjugated goat antibodies to mouse IgG. Alkaline phosphatase activity was measured as described above. Cells were observed microscopically for penetration and localization of recombinant Fv-FOXP3. Controls included medium and equimolar amounts of Fv and FOXP3 alone. Control medium consisted of McCoy's medium containing 5\% fetal calf serum, 5\% glycerol, and $5 \mathrm{mM}$ imidazole similar to the composition of purified Fv-FOXP3 and FOXP3 after exchange dialysis. In order to determine if intracellular Fv-FOXP3 was full-length, SKOV-3 cells were incubated with Fv-FOXP3 in 6-well plates, washed twice with PBS, and lysed with $10 \%$ SDS. Aliquots of cell lysate were electrophoresed in 4-20\% gradient SDS-PAGE, and Fv-FOXP3 was identified by Western blotting to nitrocellulose membranes developed with anti-FOXP3 antibodies as above.

Fv-FOXP3 cytotoxicity in vitro. Cancer cells were grown in medium with $10 \%$ FCS in the presence of $5 \% \mathrm{CO}_{2}$. Cells were added to each of multiple wells in 96-well plates and grown to $40 \%$ confluence overnight. Recombinant Fv, FOXP3, Fv-FOXP3 dialyzed against McCoy's medium, and medium control were added undiluted to wells at $10 \mu \mathrm{g} / \mathrm{ml}$ and in serial dilutions. Cell death was measured by the nuclear uptake of propidium iodide and expressed as the mean percent \pm SD by counting 100 cells in 3 separate areas of the wells.

Apoptosis assays. MCF-7 cells were grown to confluence in T-25 flasks and incubated with Fv-FOXP3 at concentrations that resulted in cell death by $24 \mathrm{~h}$, but at which cell death was not observable within $5 \mathrm{~h}$. An apoptosis positive control was performed by heat shocking cells at $45^{\circ} \mathrm{C}$ for $20 \mathrm{~min}$. Cell lysates were prepared with $0.5 \mathrm{ml}$ RIPA buffer (Pierce Chemical Co.) containing HALT protease inhibitor cocktail (Pierce) or by solubilization in $10 \%$ SDS. Protein $(20 \mu \mathrm{g} /$ lane) from cell lysates was evaluated by SDS-PAGE and transblotted to PVDF membranes. Western blots were performed with primary antibodies incubated overnight against activated caspase-3 p17 cleavage peptide and actin loading control. Blots were incubated with HRP-conjugated secondary antibodies at 1:2000 dilution for $1.5 \mathrm{~h}$, washed three times with TBST, then incubated with enhanced chemiluminescence (ECL) detection system (Amersham Pharmacia, Buckinghamshire, UK) and exposed to X-OMAT film (Eastman Kodak, Rochester, NY). Cell death in response to caspase inhibition was evaluated in MCF-7, MDA-MB-231, SKOV-3, and OVCAR-3 cells with the general caspase inhibitor, Z-VADFMK (Alexis Biochemicals, San Diego, CA). Cells were grown to $40 \%$ confluence in 96 -well plates and preincubated for $1 \mathrm{~h}$ with $20 \mu \mathrm{M}$ Z-VAD-FMK or control medium and then exposed to Fv-FOXP3. Cell death was determined by the nuclear uptake of propidium iodide $18 \mathrm{~h}$ after addition of Fv-FOXP3.

Fv-FOXP3 cytotoxicity in vivo. A 'hemispleen' model, as first described by Schulick et al (7) was optimized as previously described (6). Balb/c mice at 10 weeks of age were purchased from the Jackson Laboratory (Bar Harbor, $\mathrm{ME}$ ) and injected with $3.5 \times 10^{5}$ CT26 colon cancer cells into the inferior hemispleen. The inferior hemispleen was then removed. Ten minutes later, the treatment or control solution was injected into the superior hemispleen. The hemispleen was left in place for a second injection 7 days later. The animals were euthanized 2 weeks later, and the livers were 
A.

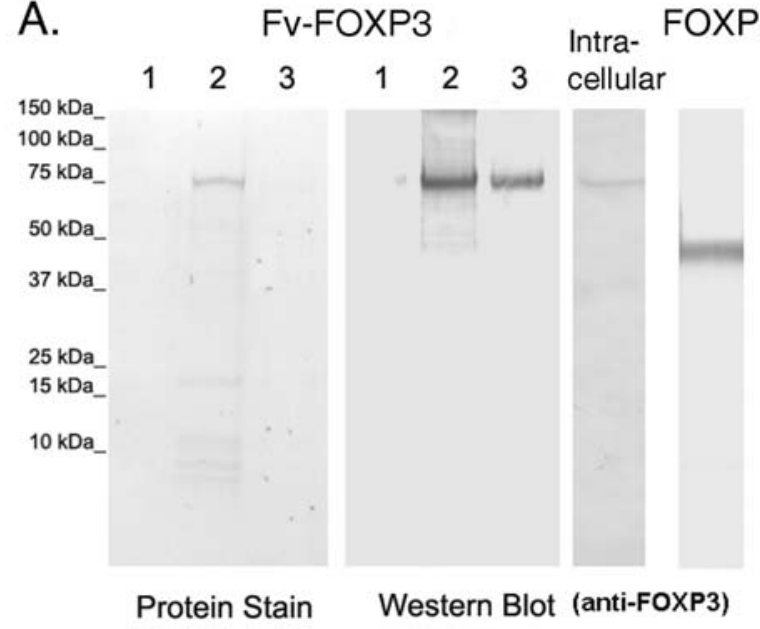

B.

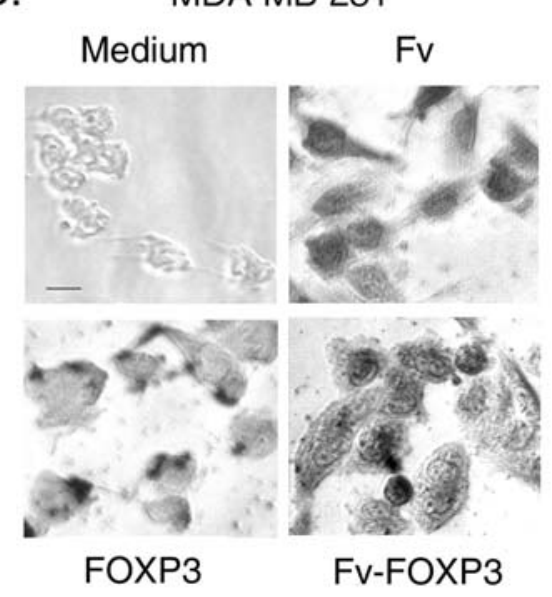

Figure 1. Fv-FOXP3 isolation and penetration in cancer cells. A, Fv-FOXP3 isolated from X-33 cell lysate by IMAC and characterized by SDS-PAGE and Western blotting. Lane 1 represents the wash fraction, and lanes 2 and 3 eluted fractions. The predominant eluted protein of $75 \mathrm{kDa}$ is consistent with full length Fv-FOXP3 as confirmed in the Western blotting developed with antibodies to FOXP3. Intracellular full length Fv-FOXP3 is shown in Western blotting from lysate of SKOV-3 cells incubated with FV-FOXP3 and compared with FOXP3 (47 kDa) alone. B, Cellular penetration of Fv-FOXP3. Fv-FOXP3 localized in the nucleus and cytoplasm of MDA-MB-231 cells similar to Fv alone. By contrast, FOXP3 alone did not show nuclear localization. Cells incubated with Fv-FOXP3 and FOXP3 were developed with antibodies to FOXP3, whereas cells incubated with Fv alone were developed with antibodies to myc. Cells incubated with medium alone did not stain for FOXP3. Bar, $10 \mu \mathrm{m}$.

examined. The whole liver was assigned a metastasis score of 0 (no gross metastasis), 1 ( $<1 \mathrm{~cm}^{2}$ area of tumor), $2\left(1-2 \mathrm{~cm}^{2}\right.$ area of tumor), 3 ( $>2 \mathrm{~cm}^{2}$ area of tumor), or 4 (complete infiltration) as previously described (6). These studies were reviewed and approved by the Animal Review Committee, Loma Linda VA Medical Center.

Statistical analyses. A P-value was determined by using a two-tailed Student's t-test.

\section{Results}

An Fv-FOXP3 fusion protein penetrates into cancer cells. Fv-FOXP3 was isolated from X-33 cell lysate by IMAC on NiNTA agarose. SDS-PAGE analysis of eluted fractions showed a major protein band at $75 \mathrm{kDa}$ consistent with fulllength Fv-FOXP3, and Western blot analysis with antibodies to FOXP3 confirmed the identity of the recombinant protein (Fig. 1A). Lane 1 contains the wash fraction, and lanes 2 and 3 are serial eluted fractions. Fv-FOXP3 was incubated with MDA-MB-231 cells to assess cellular penetration and localization. Fv-FOXP3 localized in the nucleus and cytoplasm similar to the Fv fragment alone. By contrast, FOXP3 alone did not show nuclear localization (Fig. 1B). Localization of FOXP3 in cytoplasm is consistent with endocytosis of FOXP3 aggregates. In order to determine if Fv-FOXP3 was internalized intact, SKOV-3 ovarian cancer cells were incubated with Fv-FOXP3, washed, lysed with SDS and analyzed by SDS-PAGE and Western blotting. Full-length intracellular Fv-FOXP3 (75 kDa) was identified in Western blotting with antibodies to FOXP3 and compared to FOXP3 (47 kDa) alone (Fig. 1A).

Fv-FOXP3 kills breast and ovarian cancer cells in vitro. Cytotoxicity of Fv-FOXP3 was assessed by incubation with cancer cell lines SKOV-3, MCF-7, MDA-MB-231, and
OVCAR-3. Fv-FOXP3 $(10 \mu \mathrm{g} / \mathrm{ml})$ consistently induced cell death that was observed as early as $1 \mathrm{~h}$ and was maximal by 24 h. Cytotoxicity in response to Fv-FOXP3 was compared to a dialysate control. The mean results \pm SD of 18 experiments are shown in Fig. 2A. In six experiments, Fv-FOXP3 killed $95.3 \pm 5.9 \%$ of SKOV -3 cells compared to $1.0 \pm 0.0 \%$ of controltreated cells $\left(\mathrm{P}<1.0 \times 10^{-6}\right)$. In five experiments, FV-FOXP3 killed $91.6 \pm 8.0 \%$ of MCF-7 cells compared to $1.0 \pm 0.0 \%$ of control-treated cells $\left(\mathrm{P}<1.0 \times 10^{-6}\right)$. In four experiments, FvFOXP3 killed $89.5 \pm 16.5 \%$ of MDA-MB-231 cells compared to $2.0 \pm 0.0 \%$ of control-treated cells $\left(\mathrm{P}<1.0 \times 10^{-3}\right)$. In three experiments, Fv-FOXP3 killed $82.7 \pm 17.5 \%$ of OVCAR-3 cells compared to $4.3 \pm 0.6 \%$ of control-treated cells $\left(\mathrm{P}<1.0 \times 10^{-3}\right)$. In two separate experiments, the mean cytotoxicity of the four cancer cell lines in response to $\mathrm{Fv}$ alone $(10 \mu \mathrm{g} / \mathrm{ml})$ and FOXP3 alone $(10 \mu \mathrm{g} / \mathrm{ml})$ was $2.3 \pm 1.3$ and $1.8 \pm 0.2$, respectively. These results demonstrate that cytotoxicity was a result of Fv-FOXP3 and not the antibody Fv fragment or FOXP3 alone. Fig. 2B is an example of cells stained with propidium iodide and shows that there was no increase in cell death above the dialysate control with Fv or FOXP3 alone tested at the same concentrations as Fv-FOXP3.

Dose-dependent killing of cancer cells by Fv-FOXP3. In order to evaluate a dose-dependent effect of Fv-FOXP3, cancer cells MCF-7, MDA-MB-231, OVCAR-3, and SKOV-3 were incubated with FV-FOXP3 and comparable concentrations of Fv and FOXP3 alone at serial dilutions for $24 \mathrm{~h}$. Cell death was not observed in response to Fv and FOXP3 alone. By contrast, dose-dependent cell death was observed in response to Fv-FOXP3. Fig. 3 shows a representative example of three separate experiments. Fv-FOXP3 killed cells within 1-4 h at the highest concentration $(10 \mu / \mathrm{ml})$. Cell cultures were monitored for 3 days, and maximum cytotoxicity of FV-FOXP3 was observed by $24 \mathrm{~h}$ at all concentrations tested (data not shown). 
A.

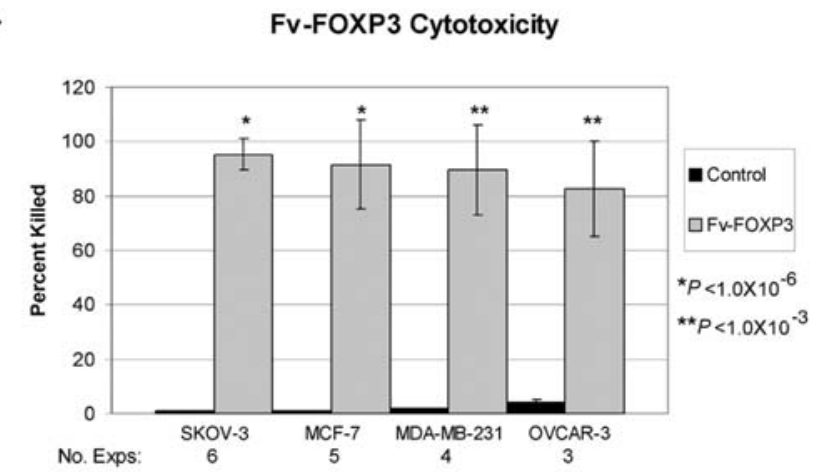

B.

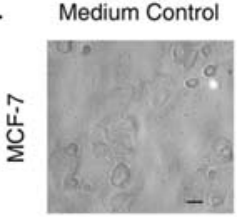

Fv

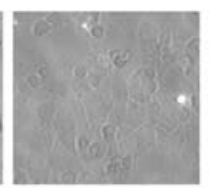

FOXP3

Fv-FOXP3
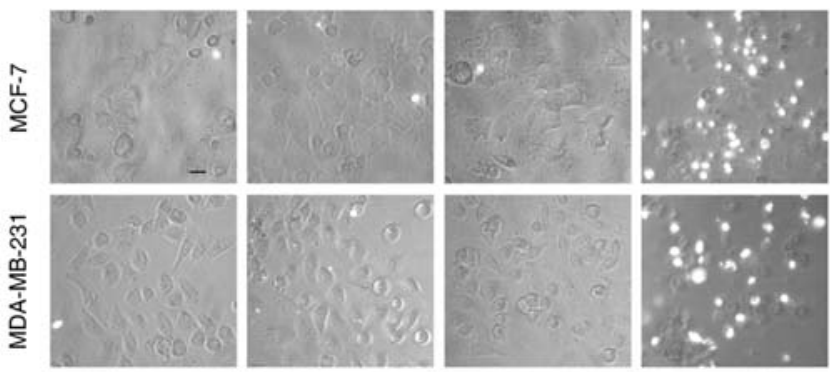

के
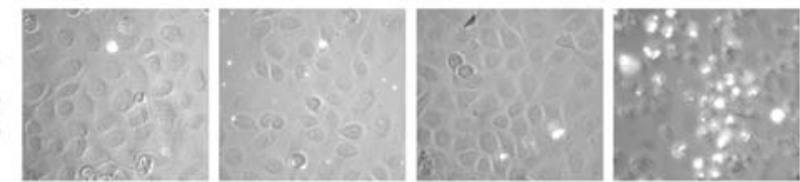

Figure 2. Fv-FOXP3 kills breast and ovarian cancer cell lines in vitro. A, FvFOXP3 $(10 \mu \mathrm{g} / \mathrm{ml})$ incubated for $24 \mathrm{~h}$ with the breast cancer cell lines MCF-7 and MDA-MB-231, and ovarian cancer cell lines SKOV-3 and OVCAR-3 demonstrated marked cytotoxicity compared to a dialysate control. Fv alone and FOXP3 alone were not cytotoxic (data presented in the text). Results are expressed as mean percent killed \pm SD. B, Propidium iodide staining of cancer cells after incubation with medium (control dialysate), Fv alone, FOXP3 alone, and Fv-FOXP3 for $24 \mathrm{~h}$. The results show that Fy and FOXP3 alone were not cytotoxic. Bar, $20 \mu \mathrm{m}$.

\section{Fv-FOXP3 Dose-Response}

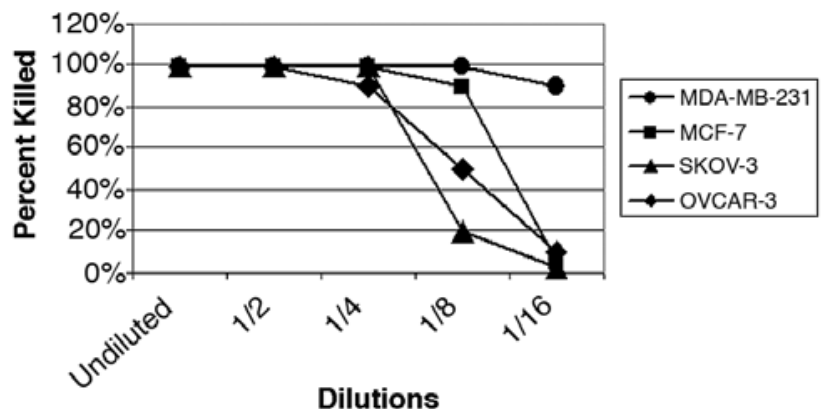

Figure 3. Dose-dependent killing of cancer cells by Fv-FOXP3. Fv-FOXP3 was incubated at serial dilutions with breast and ovarian cancer cell lines for $24 \mathrm{~h}$. Cell death was determined by the nuclear uptake of propidium iodide. Results are expressed as mean percent killed \pm SD.

Fv-FOXP3 induces cell death by apoptosis. Studies were performed to determine the mechanism of cell death in response to Fv-FOXP3. MDA-MB-231, MCF-7, SKOV-3,
A.

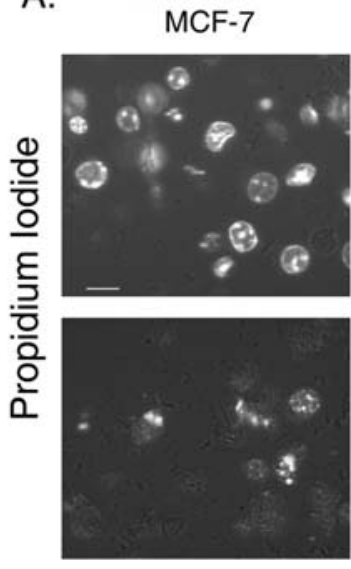

MDA-MB-231

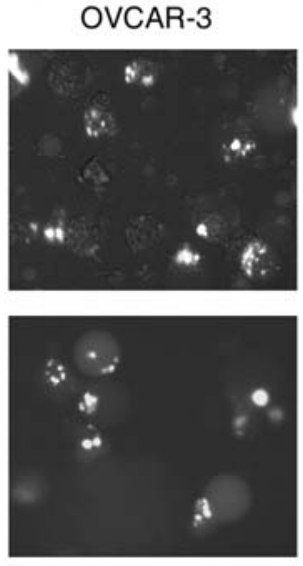

SKOV-3
B.

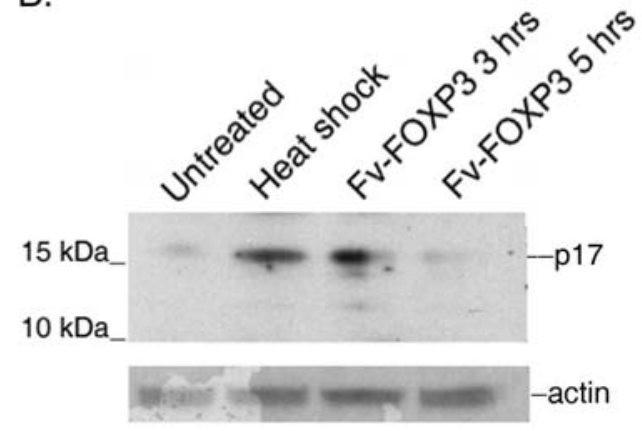

MCF-7 Cells

Figure 4. Fv-FOXP3 induces cell death by apoptosis. A, The pattern of nuclear uptake of propidium iodide in breast and ovarian cancer cell lines treated with Fv-FOXP3 shows evidence of apoptosis. MDA-MB-231, MCF-7, SKOV-3, and OVCAR-3 cancer cell lines were incubated with Fv-FOXP3 for $4 \mathrm{~h}$, stained with propidium iodide, and observed microscopically. Cells demonstrate nuclear fragmentation and peripheral chromatin condensation characteristic of early apoptosis. Bar, $20 \mu \mathrm{m}$. B, Lysates of MCF-7 cells after treatment with Fv-FOXP3 contain p17 activated fragment of caspase-3 as shown by Western blotting. Untreated cells and heat shock cells were used as negative and positive controls, respectively.

and OVCAR-3 cancer cells were incubated with Fv-FOXP3, stained with propidium iodide, and visualized microscopically. Cells showed nuclear fragmentation and peripheral chromatin condensation characteristic of apoptosis (Fig. 4A). Since the apoptosis mechanism is mainly caspase-dependent, a Western blot assay was used to detect the p17 activated fragment of caspase-3 as further evidence of apoptosis. Cell lysates from MCF-7 cells contained the p17 activated fragment of caspase-3 after treatment with Fv-FOXP3 consistent with induction of apoptosis (Fig. 4B). Untreated cells served as a negative control and heat shock cells as a positive control. Further confirmation of apoptosis was obtained by inhibiting cell death in response to Fv-FOXP3 by the general caspase inhibitor, Z-VAD-FMK. A representative example of three experiments is shown in Fig. 5A. Z-VAD-FMK reduced Fv-FOXP3-mediated killing of SKOV-3 (92.5 $\pm 4.5 \%$ to $6.0 \pm 1.4 \%, \mathrm{P}=0.001), \mathrm{MCF}-7(87.5 \pm 3.5$ to $25.0 \pm 4.5 \%$, $\mathrm{P}=0.01)$, MDA-MB-231 cells $(62.5 \pm 3.5 \%$ to $6.0 \pm 3.5 \%$, 
A.

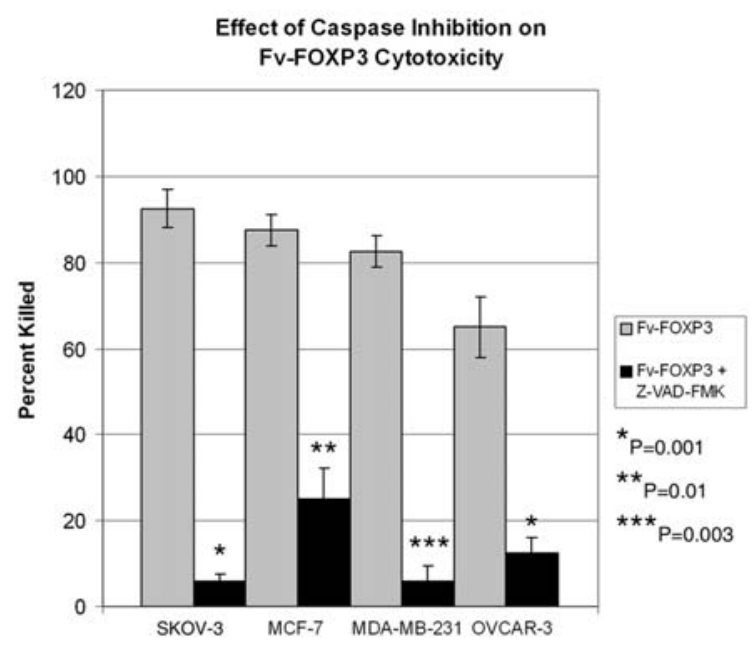

B.

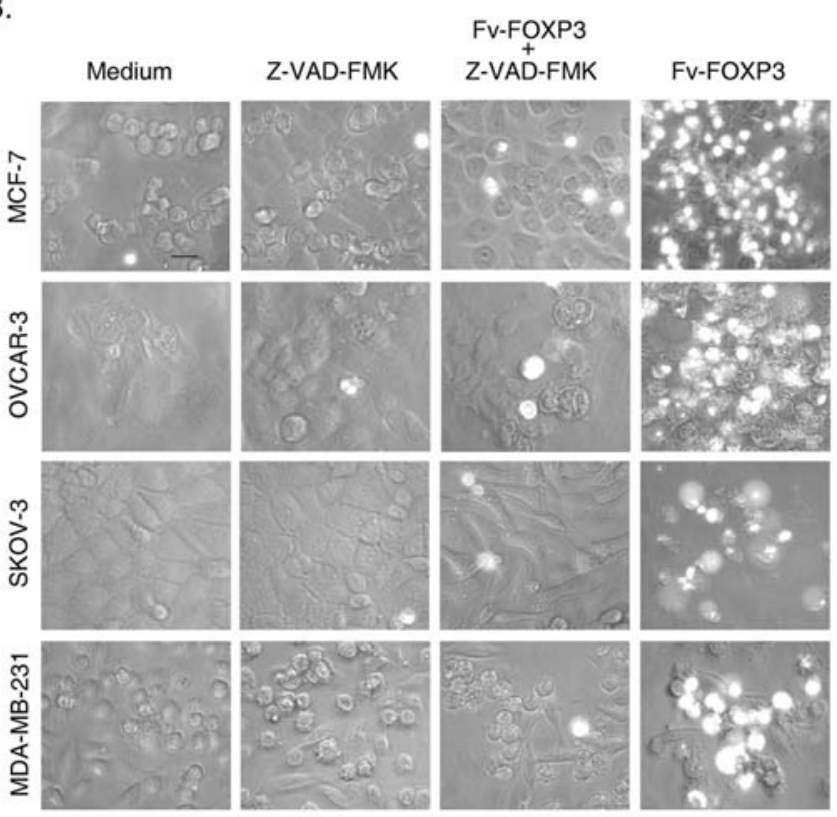

Figure 5. Inhibition of Fv-FOXP3 cytotoxicity by Z-VAD-FMK caspase inhibitor. A, SKOV-3, MCF-7, MDA-MB-231, and OVCAR-3 were incubated with Fv-FOXP3 alone and in the presence of Z-VAD-FMK. Significant inhibition of Fv-FOXP3 cytotoxicity was observed in the presence of Z-VADFMK. Results are expressed as mean \pm SD. B, Propidium iodide staining of cancer cells treated with control medium, Z-VAD-FMK alone, Fv-FOXP3 with Z-VAD-FMK, and Fv-FOXP3 alone. Z-VAD-FMK inhibited cytotoxicity in response to Fv-FOXP3. Bar, $20 \mu \mathrm{m}$.

$\mathrm{P}=0.003)$, and OVCAR-3 $(65.0 \pm 7.1 \%$ to $12.5 \pm 3.5 \%, \mathrm{P}=0.01)$. Representative results of cells stained with propidium iodide are shown in Fig. 5B. Z-VAD-FMK was then studied for any possible effect on cellular penetration by Fv-FOXP3. Fv-FOXP3 penetrated MDA-MB-231 cells comparably in the absence or presence of $20 \mathrm{mM} \mathrm{Z-VAD-FMK} \mathrm{(data} \mathrm{not}$ shown). Therefore, inhibition of cell death in response to FV-FOXP3 could not be attributed to inhibition of cellular penetration by Fv-FOXP3. It appears from our studies that apoptosis participates as a mechanism of cell death in response to Fv-FOXP3.

Fv-FOXP3 kills colon cancer cells in vitro and inhibits development of colon cancer metastasis in vivo. After demonstrating the cytotoxicity of Fv-FOXP3 for breast and ovarian cancer cells, we next tested its effect on CT26 colon cancer cells in vitro. In five experiments, Fv-FOXP3 killed $99.0 \pm 2.2 \%$ of CT26 cells compared to $2.0 \pm 2.0 \%$ of controltreated cells $(\mathrm{p}<0.001)$ (Fig. 6A), indicating the susceptibility of the CT26 cell line to Fv-FOXP3 therapy. A syngeneic liver metastasis model, generated by injecting CT26 colon carcinoma cells into Balb/c mice, was then used to test the efficacy of Fv-FOXP3 protein therapy in vivo. In a comparison of 8 control and 8 treated mice, Fv-FOXP3 reduced the metastasis score from $3.6 \pm 0.2$ to $3.0 \pm 0.2$ ( $\mathrm{P}=0.03$ ) (Fig. 6B). The reduction in metastasis score represents a clinically significant reduction in tumor burden as indicated by the metastatic score criteria described in Materials and methods and in Hansen et al (6). Moreover, systemic administration of the Fv-FOXP3 fusion protein was not associated with any significant toxicity other than mild scar formation in the spleens at the site of injection. The absence of systemic toxicity and demonstrated efficacy of Fv-FOXP3 protein therapy in vivo indicate the clinical potential of antibodymediated FOXP3 protein replacement therapy.

\section{Discussion}

FOXP3 was initially described as a unique marker of CD25+ T-regulatory cells, and absence of its expression was associated with development of autoimmunity (8). Subsequent studies have elucidated a role of FOXP3 in tumor suppression $(5,9)$, and augmentation of FOXP3 function is, therefore, of interest as a potential therapy for cancer. FOXP3 gene therapy may have clinical utility in the future, but gene therapy is presently hindered by technical limitations.

The cell-penetrating antibody, mAb 3E10 and its Fv fragment, have been developed as transport vehicles capable of intranuclear delivery of therapeutic molecules into living cells $(6,10)$, and antibody-mediated protein transduction is an alternative to gene therapy. mAb $3 \mathrm{E} 10 \mathrm{Fv}$ was shown to penetrate living cells by a novel mechanism involving the ENT2 nucleoside salvage pathway (11), and penetration of $\mathrm{mAb} 3 \mathrm{E} 10$ into the nucleus of living cells is consistent with this finding in contrast to other cell-penetrating antibodies internalized into endosomes through lipid rafts. We have previously shown that 3E10 Fv transported wild-type p53 into cancer cells in vitro and in vivo, and Fv-p53 was cytotoxic to numerous cancer cell lines. In addition, Fv-p53 protein therapy inhibited development of colon cancer metastasis to liver in vivo in a mouse model of colon cancer metastasis (6). Based on our previous success in using $3 \mathrm{E} 10 \mathrm{Fv}$ to transport p53 into cancer cells, we sought to determine if the $3 \mathrm{E} 10 \mathrm{Fv}$ would also mediate FOXP3 protein therapy.

In the present study we have demonstrated that the fusion protein, Fv-FOXP3, penetrates cancer cell lines and localizes in the cell nucleus. Moreover, Fv-FOXP3 induces apoptosis in various cancer cell lines in vitro as shown by the appearance of apoptotic nuclei, the presence of the activated fragment of caspase- 3 by Western blotting, and inhibition of apoptosis by the general caspase inhibitor, Z-VAD-FMK. Apoptosis in response to Fv-FOXP3 was dose-dependent, occurred as early as $1 \mathrm{~h}$ and was maximal by $24 \mathrm{~h}$. Treatment with FvFOXP3 resulted in a clinically significant reduction of tumor 
A.

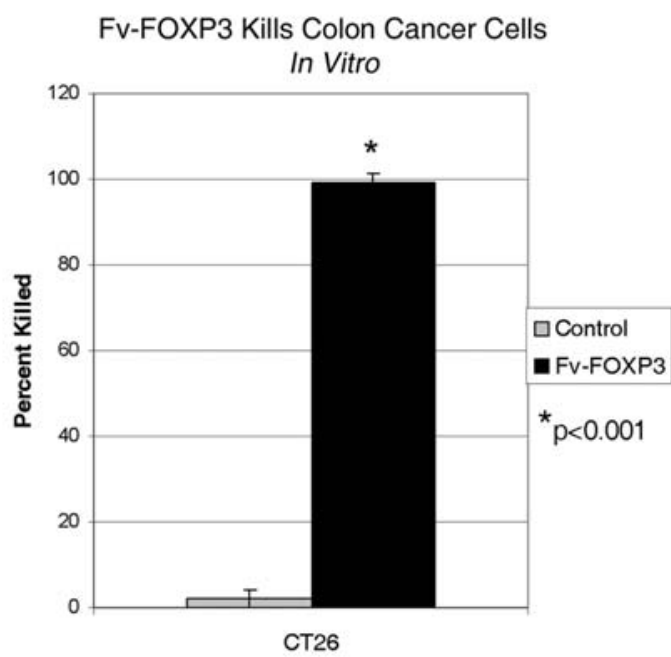

Number of experiments $=5$
B.

Effect of Fv-FOXP3 on Development of Liver Metastasis

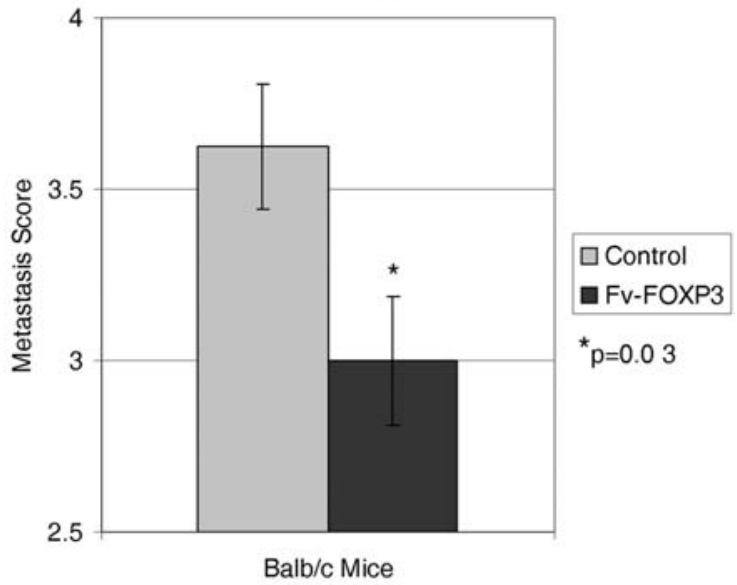

Number of animals in each group $=8$

Figure 6. Fv-FOXP3 kills colon cancer cells in vitro and inhibits development of colon cancer metastasis in vivo. A, Fv-FOXP3 kills CT26 colon cancer cells in vitro. CT26 colon cancer cells were incubated for $24 \mathrm{~h}$ with Fv-FOXP3. Cell death was measured by the nuclear uptake of propidium iodide. Results are expressed as mean $\pm \mathrm{SD}$. B, Fv-FOXP3 inhibits development of liver metastasis of CT26 colon cancer cells in a syngeneic model of liver metastasis in Balb/c mice. Control mice were treated with dialysate alone. Metastasis score is defined as follows: 0 (no gross metastasis), $1\left(<1 \mathrm{~cm}^{2}\right.$ area of tumor), $2\left(1-2 \mathrm{~cm}^{2}\right.$ area of tumor), 3 ( $>2 \mathrm{~cm}^{2}$ area of tumor), or 4 (complete infiltration). Results are expressed as mean \pm SEM and demonstrate clinically significant reduction in tumor burden.

burden of colon cancer cells in vivo in an animal model of colon cancer metastasis to liver. The finding that Fv-FOXP3 inhibited cancer metastasis without evidence of systemic toxicity is clinically important.

The identification of FOXP3 as a tumor suppressor protein has generated significant interest in the development of FOXP3 as a therapeutic agent and established the need for an effective means of delivery of FOXP3 into cell nuclei. We have now shown that the $3 \mathrm{E} 10 \mathrm{Fv}$ fragment is capable of mediating FOXP3 protein therapy both in vitro and in vivo, and we anticipate that Fv-FOXP3 protein therapy will find numerous applications since FOXP3 appears to be critically involved in a multitude of biochemical pathways. FOXP3 has known roles in immune suppression. In tumor suppression FOXP3 was initially shown to inhibit the expression of the HER-2 oncogene (5). However, FOXP3 also inhibited cancer cells that did not over-express HER-2. Subsequently, FOXP3 was shown to inhibit expression of other oncogenes such as SKP2, thus explaining its effect on different cancer cells lines (12). Our present results confirm the findings of Zuo et al that FOXP3 is cytotoxic for breast cancers as well as those originating from other tissues, and implicate multiple mechanisms responsible for FOXP3 function in cancer cells.

Our studies highlight the role of FOXP3 protein replacement therapy as an alternative to gene replacement in cancer therapy. Future studies will focus on investigating the full clinical potential of Fv-FOXP3 protein therapy as a treatment for cancer, the ability of FV-FOXP3 to modulate immune activity, and testing Fv-FOXP3 protein replacement therapy as a potential treatment for the IPEX syndrome. As a greater understanding of the biochemical function of FOXP3 is obtained, it is likely that even further applications of Fv-FOXP3 protein therapy will be identified.

\section{Acknowledgments}

This study was supported by a grant from the Veterans Affairs (R.H. Weisbart) and a seed grant from the Department of Surgery, Loma Linda University (M.E. Reeves). We thank Michael Cho for technical assistance and Dr Joseph Gera for critical reading of the manuscript. E.H. and S.C. are co-first authors; R.N., M.R., and R.W. are co-senior authors.

\section{References}

1. Brunkow ME, Jeffery EW, Hjerrild KA, et al: Disruption of a new forkhead/winged-helix protein, scurfin, results in the fatal lymphoproliferative disorder of the scurfy mouse. Nat Genet 27: 68-73, 2001.

2. Schubert LA, Jeffery E, Zhang Y, Ramsdell F and Ziegler SF: Scurfin (FOXP3) acts as a repressor of transcription and regulates T cell activation. J Biol Chem 276: 37672-37679, 2001.

3. Wildin RS, Ramsdell F, Peake J, et al: X-linked neonatal diabetes mellitus, enteropathy and endocrinopathy syndrome is the human equivalent of mouse scurfy. Nat Genet 27: 18-20, 2001.

4. Bennett CL, Christie J, Ramsdell F, et al: X-linked neonatal diabetes mellitus, enteropathy and endocrinopathy syndrome is the human equivalent of mouse scurfy. Nat Genet 27: 20-21, 2001.

5. Zuo T, Wang L, Morrison C, et al: FOXP3 is an X-linked breast cancer suppressor gene and an important repressor of HER-2/ ErbB2 oncogene. Cell 129: 1275-1286, 2007.

6. Hansen JE, Fischer LK, Chan G, et al: Antibody-mediated p53 protein therapy prevents liver metastasis in vivo. Cancer Res 67: 1769-1774, 2007

7. Jain A, Slansky JE, Matey LC, Allen HE, Pardol DM and Schulick RD: Synergistic effect of a granulocyte-macrophage colony-stimulating factor-transduced tumor vaccine and systemic interleukin-2 in the treatment of murine colorectal cancer hepatic metastases. Ann Surg Oncol 10: 810-820, 2003. 
8. Hori S, Nomura T and Sakaguchi S: Control of regulatory T cell development by the transcription factor Foxp3. Science 299: 1030-1031, 2003.

9. Chen G-Y, Chen C, Wang L, Chang X, Zheng P and Liu Y: Broad expression of the FoxP3 locus in epithelial cells: a caution against early interpretation of fatal inflammatory diseases following in vivo depletion of FoxP3-expressing cells. J Immunol 180: 5163-5166, 2008.

10. Weisbart RH, Stempniak M, Harris S, Zack DJ and Ferreri K: An autoantibody is modified for use as a delivery system to target the cell nucleus: therapeutic implications. J Autoimmun 11: 539-546, 1998
11. Hansen JE, Tse CM, Chan G, Heinze ER, Nishimura RN and Weisbart RH: Intranuclear protein transduction through a nucleoside salvage pathway. J Biol Chem 282: 20790-20793, 2007.

12. Zuo T, Liu R, Zhang H, et al: FOXP3 is a novel transcriptional repressor for the breast cancer oncogene SKP2. J Clin Invest 117: 3765-3773, 2007 